\title{
Anorectal Malformation: An Atypical Association of Pierre Robin Sequence
}

\author{
Manal M. Farouk ${ }^{1 *}$, Sherif N. Kaddah', Magd Ahmed Kotb² \\ 1 Department of Pediatric Surgery, Faculty of Medicine, Cairo University, Egypt; \\ sherifkaddah@kasralainy.edu.eg \\ 2 Department of Pediatrics, Faculty of Medicine, Cairo University, Egypt; \\ magdkotb@kasralainy.edu.eg \\ * Correspondence: manal.m.mahmoud93@gmail.com \\ Received: 17/10/2021; Accepted: 26/12/2021; Published online: 27/12/2021.
}

\begin{abstract}
:
Isolated Pierre Robin sequence (PRS) is characterized by underdeveloped jaw, backward displacement of the tongue and upper airway obstruction with or without cleft palate or be part of a known syndrome as VACTREL or CHARGE, or be associated with other malformations as congenital heart disease, lung malformations, musculoskeletal system anomalies, and hereditary progressive arthro-ophthalmopathy. We report a neonate with PRS who presented by choanal atresia, sepsis, single ventricle, large ventricular septal defect; large patent ductus arteriosus, anorectal malformation in the form of high imperforate anus and rectovesical fistula. Anorectal malformation was not previously reported in Pierre Robin sequence. We report that single ventricle and anorectal malformation are rare associations of PRS. Examination for anorectal malformation, prompt diagnosis and initiation of specific management is life-saving in any neonate and especially in those with structural malformations or deformities of PRS.
\end{abstract}

Level of Evidence of Study: IV (1).

Keywords: Pierre robin sequence; anorectal malformation; ventricular septal defect; congenital heart disease; rectovesical fistula.

Abbreviations: PRS: Pierre Robin sequence.

\section{Introduction}

Pierre Robin sequence (PRS) is characterized by a small lower jaw and displacement of the tongue toward the back of the oral cavity (glossoptosis), with or without cleft palate. It may be isolated or associated with other known typical syndrome or associated with other random anomalies and malformations (2). PRS was reported to result from multiple causes, yet at many times it is indeterminate. Deletions and translocations are the commonest reported genetic abnormalities, followed by mutations of SOX9 gene in children with PRS (3). PRS is also reported to be a developmental consequence of oligohydramnios, or embryonic defect of morphogenesis (4).

PRS might be associated with other syndromes in a third of cases, as CHARGE syndrome (5), Stickler syndrome(6), campomelic dysplasia, VACTREL(7), and trisomy 11q (8).

PRS might be associated with other malformations, whereas up to $41 \%$ have cardiac defects as ventricular septal defects, and others, and up to $36 \%$ have neurologic anomalies as hypoplastic corpus callosum (9), cerebellar hypoplasia and mental retardation (10). Rare associations of PRS with other anomalies as caudal regression were also reported (11). The purpose of this work was to report atypical association of PRS and the course of management.

\section{Case Presentation}

We report a $2.5 \mathrm{~kg}$ full term male, who was born by cesarian section. The pregnancy was uncomplicated apart from polyhydramnios. The boy presented by upper airway obstruction due to bilateral choanal atresia, that necessitated immediate emergency insertion of bilateral nasopharyngeal tubes to secure airway (Figure 1). He was noted to have PRS (micrognathia, glossoptosis, cleft palate), associated with cardiac anomalies in the form of single atrium with 
unprotected pulmonary circulation, receiving both superior and inferior vena cava, and cardiac type total anomalous pulmonary venous return with 2 pulmonary veins draining in the coronary sinus that drain in the common atrium. All venous flow drained through mitral valve to a large dominant left ventricle. The tricuspid valve was atretic with rudimentary right ventricle connected to left ventricle by large ventricular septal defect. He had a large patent ductus arteriosus. He suffered from heart failure and received anti-failure medications, and fluid restriction. The boy suffered from sepsis that needed neonatal intensive care admission.
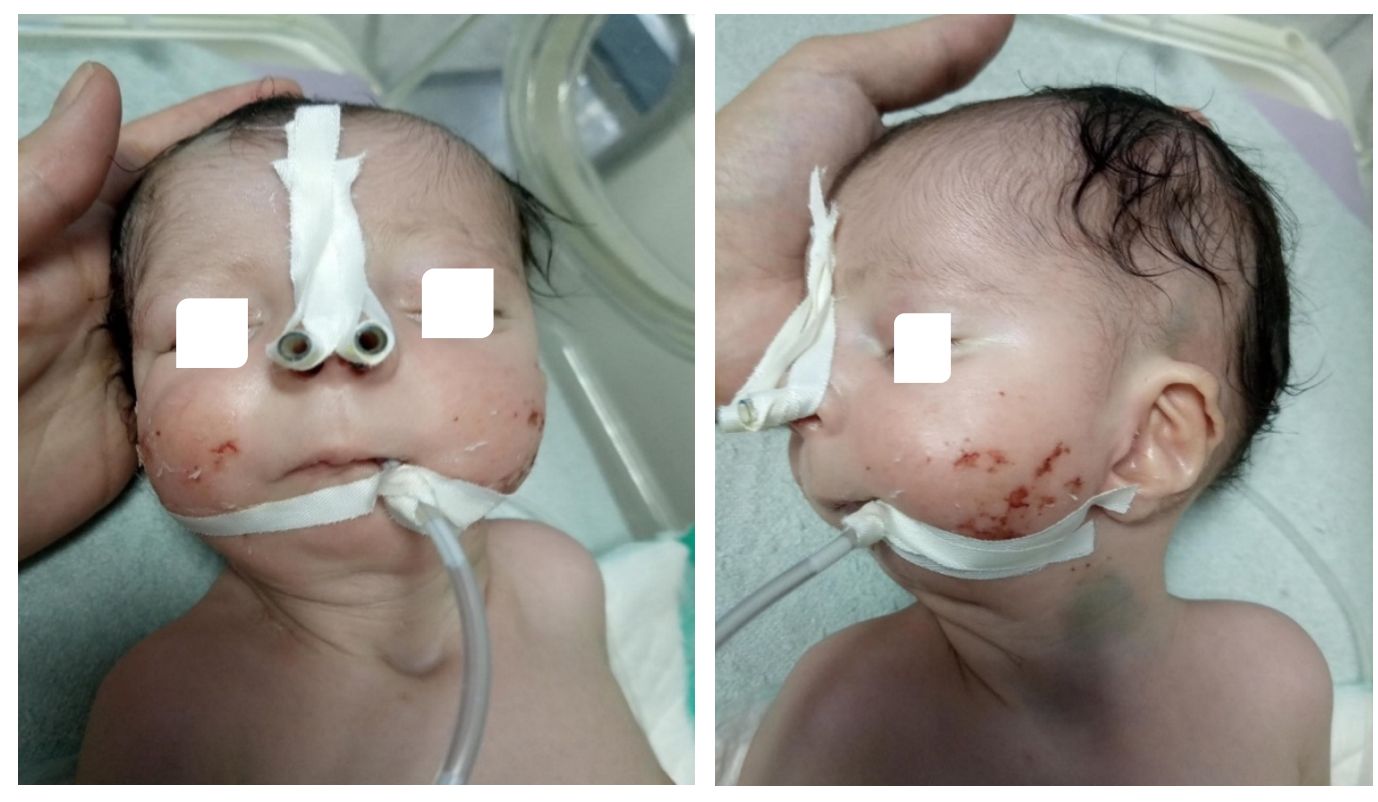

Figure 1: Facial features and Choanal stents in the new born with Pierre Robin Sequence.

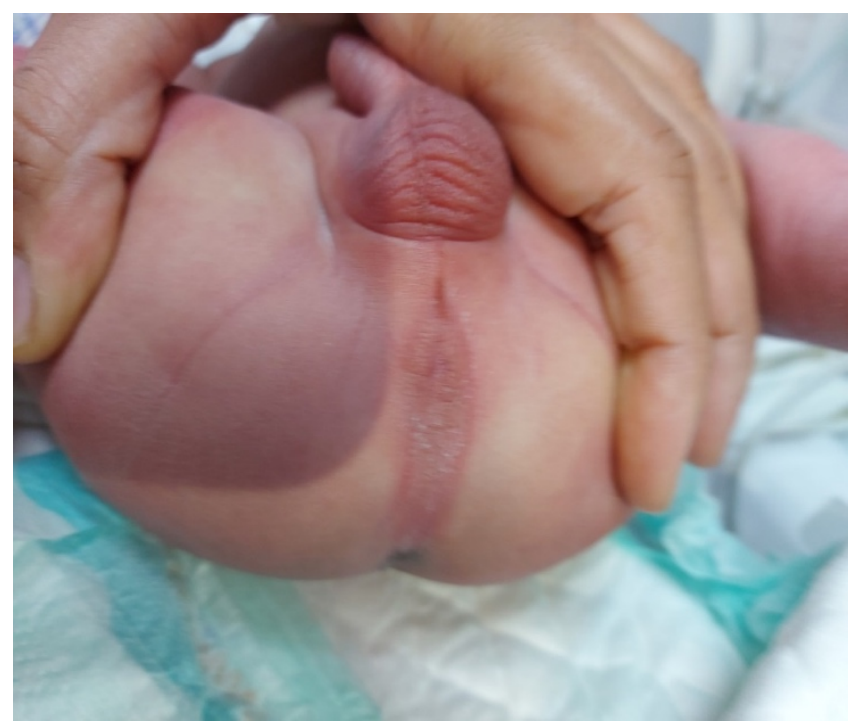

Figure 2: Anorectal malformation in a new born with Pierre Robin Sequence.

The boy did not pass stools and was referred to our unit by the age of 10 days. His weight was $2.5 \mathrm{~kg}$, length $51 \mathrm{~cm}$ and head circumference of $35 \mathrm{~cm}$. He had high imperforate anus with rectovesical fistula (Figure 2). His penis was stained with meconium. Eyes examination and transcranial ultrasound revealed no abnormalities. Abdominal sonography revealed stationary marginal bilateral pelvic dilatation on both kidneys. By next day he underwent surgical anoplasty of the high imperforate of and simple loop colostomy. Ryle tube feeding was initiated following failed oral feeding due to his underdeveloped mandible \& cleft palate. He tolerated full 
feeds within 48 hours and was discharged. A month later he presented by sepsis, multiple organ failure and died.

\section{Discussion}

We report a newborn with Pierre Robin sequence, that was associated with very serious life threatening anomalies. The boy had receding small lower jaw, glossoptosis, cleft palate, cardiac anomalies, choanal atresia and anorectal malformations. There was no eye, vertebral, joint, central nervous system, or other anomalies. His abdominal sonography revealed stationary marginal bilateral pelvic dilatation of both kidneys that necessitated follow up.

The associations were not congruent with the previously known syndromes as; CHARGE syndrome with choanal atresia, heart defects, coloboma of eye, ear abnormalities, genital abnormalities, and growth retardation (5), Stickler syndrome (6) the hereditary progressive arthro-ophthalmopathy, campomelic dysplasia, VACTREL with vertebral anomalies, anal atresia, cardiac defects, tracheo-esophageal fistula, renal defects, and limb anomalies (7), Treacher-Collins syndrome (9), trisomy 11q (8) and others (9). The overlap is a recognized feature among those with PRS (12).

The boy suffered from membranous choanal atresia necessitating immediate postnatal emergency tube insertion. He suffered from hemodynamically unstable multiple congenital heart defects that needed immediate attention and anti-failure measures. His condition was further complicated by septicemia. The stabilization of the cardiac condition and control of sepsis were challenging, and substantially delayed the ability to refer the newborn to the neonatal surgery unit.

PRS is a clinically challenging and demanding condition. PPS is notorious for its association with patent ductus arteriosus, atrial septal defects, ventricular septal defects, and to a lesser extent complex congenital heart anomalies. Yet, it was noted that single ventricle was a rare association of PRS $(9,13)$. The boy suffered substantial feeding difficulties because of the glossoptosis, smaller lower jaw and cleft palate that precluded age appropriate weight gain.

We stress that the classical stereotyped co-existing malformations may overlap without a definite limit or exclusion.

\section{Conclusion}

Any newborn with PRS needs to be examined for all systems, and structural malformations immediately after birth, needs professional attention of a medical team. There is a need for coordinated stabilization and referral of neonates with PRS to tertiary care facility. Anorectal malformation is a very rare association, yet is a life threatening condition that should be suspected and examined for in any newborn, especially in those with associated congenital anomalies. Anorectal malformation is amenable to surgical management.

Author Contributions: All authors searched medical literature, databases, conceptualized, conducted the case review and reviewed the final manuscript. All authors have read and agreed to the published version of the manuscript.

\section{FUNDING}

Authors declare there was no extramural funding provided for this study.

\section{CONFLICT OF INTEREST}

The authors declare no conflict of interest. Authors declare veracity of information.

\section{References}

1. S. Tenny, M. Varacallo, Evidence Based Medicine. (StatPearls Publishing; Treasure Island (FL), 2020; https://www.ncbi.nlm.nih.gov/books/NBK470182/).

2. S. T. Hsieh, A. S. Woo, Pierre Robin Sequence. Clin. Plast. Surg. 46, 249-259 (2019).

3. S. Varadarajan, T. M. Balaji, A. T. Raj, A. A. Gupta, S. Patil, T. H. Alhazmi, H. A. A. Alaqi, N. E. M. Al Omar, S. A. B. A. Almutaher, A. A. Jafer, I. A. Hedad, Genetic Mutations Associated with Pierre Robin Syndrome/Sequence: A Systematic Review. Mol. Syndromol. 12, 69-86 (2021). 
4. K. N. Evans, K. C. Sie, R. A. Hopper, R. P. Glass, A. V. Hing, M. L. Cunningham, Robin sequence: from diagnosis to development of an effective management plan. Pediatrics. 127, 936-948 (2011).

5. S. J. Drew, Clefting Syndromes. Atlas Oral Maxillofac. Surg. Clin. 22, 175-181 (2014).

6. E. Pacella, A. Malvasi, A. Tinelli, F. Laterza, D. Dell'Edera, F. Pacella, F. Mazzeo, A. Ferraresi, K. G. Malarska, C. Cavallotti, Stickler syndrome in Pierre-Robin sequence prenatal ultrasonographic diagnosis and postnatal therapy: two cases report. Eur. Rev. Med. Pharmacol. Sci. 14, 1051-1054 (2010).

7. M. M. Al-Qattan, The Classification of VACTERL Association into 3 Groups According to the Limb Defect. Plast. Reconstr. Surg. - Glob. Open. 9, e3360 (2021).

8. R. Wallerstein, F. Desposito, H. Aviv, M. Schenk, D. F. Wallerstein, Partial Trisomy 11q in a Female Infant with Robin Sequence and Congenital Heart Disease. Cleft Palate. Craniofac. J. 29, 77-79 (1992).

9. R. J. H. Logjes, M. Haasnoot, P. M. A. Lemmers, M. F. A. Nicolaije, M.-J. H. van den Boogaard, A. B. Mink van der Molen, C. C. Breugem, Mortality in Robin sequence: identification of risk factors. Eur. J. Pediatr. 177, 781-789 (2018).

10. D. A. Stevenson, J. C. Carey, A novel multiple congenital anomaly-mental retardation syndrome with Pierre Robin sequence and cerebellar hypoplasia in two sisters. Am. J. Med. Genet. A. 143A, 22212226 (2007).

11. M. Ilyas, I. Bashir, I. Ellahi, F. Shaheen, N. Choh, Pierre Robin syndrome with caudal regression syndrome-a rare combination of congenital syndromes. Childs Nerv. Syst. 34, 1105-1106 (2018).

12. S. K. Jaiswal, K. K. Sukla, V. Gupta, A. K. Rai, Overlap of Patau and Pierre Robin syndromes along with abnormal metabolism: an interesting case study. J. Genet. 93, 865-868 (2014).

13. C. V. Shah, Cardiac Malformations With Facial Clefts: With Observations on the Pierre Robin Syndrome. Am. J. Dis. Child. 119, 238 (1970).

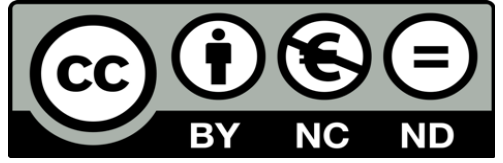

(C) 2021 submitted by the authors. Open access publication under the terms and conditions of the Creative Commons Attribution (CC- BYNC- ND) license. (https://creativecommons.org/licenses/by-nc-nd/2.0/). 